\title{
A simple AuNPs-based colorimetric aptasensor for chlorpyrifos detection
}

\section{Yuan Liu}

Taotao Li

Gaojian Yang

Yan Deng

Xianbo Mou

Nongyue He ( $\sim$ nyhe1958@163.com )

southeast university

\section{Research Article}

Keywords: Aptamer, Colorimetric aptasensor, SELEX, Chlorpyrifos, AuNPs

Posted Date: March 14th, 2022

DOl: https://doi.org/10.21203/rs.3.rs-302356/v2

License: (c) (i) This work is licensed under a Creative Commons Attribution 4.0 International License. Read Full License

Version of Record: A version of this preprint was published at Chinese Chemical Letters on November 1st, 2021. See the published version at https://doi.org/10.1016/j.cclet.2021.11.025. 


\section{Abstract}

In this work, a simple gold nanoparticles (AuNPs) based colorimetric biosensor was developed for chlorpyrifos (Chl) detection using an aptamer as the capture probe. The Chl-aptamer with low dissociation constant $(\mathrm{Kd})$ of $58.59 \pm 6.08(\mathrm{nmol} / \mathrm{L})$ was selected by ssDNA library immobilized systematic evolution of ligands by enrichment (SELEX). In the absence of Chl, the Chl-aptamer acted as the stabilizer for AuNPs in salt solution. In the presence of Chl, the highly specific Chl-aptamer bound with Chl targets immediately, thus a self-aggregation of AuNPs induced by salt was displayed. The fabricated colorimetric aptasensor exhibited an excellent sensitivity for Chl detection with the LOD as low as 14.46 $\mathrm{nmol} / \mathrm{L}$. In addition, the aptasensor was applied to test $\mathrm{Chl}$ in tap water, cucumber and cabbage samples, the excellent recoveries with acceptable RSD values below $5 \%$ demonstrated that the method can be considered as a promising tool for simple, rapid Chl detection.

\section{Full Text}

Chlorpyrifos (Chl), a typical organophosphorus pesticide, is mainly used for industry, agricultural pest control and home pests kill. The abuse of $\mathrm{Chl}$ could cause environmental pollution including water contamination, soil degradation, accumulation in crops and potential risk to animals [1-3]. Besides, exposure to $\mathrm{Chl}$ also has great threat to human health, such as affecting male reproduction [4], neurotoxicity [5], nephrotoxicity [6], inducing the proliferation of breast cancer cells $[7,8]$ and acute toxicity [9]. Thus, the toxicity of Chl to environment and humans lead to urgently exploit sensitive, simple, low cost and on-site rapid methods for $\mathrm{Chl}$ analysis and detection, since the instrumental techniques (high performance liquid chromatography [10,11], gas chromatography [12], mass spectrometry [13], surface enhanced Raman scattering [14], etc.) have the storages of bulky to carry, expensive cost, professional operators and complexity sample pre-treatment. Besides, the solution of most pesticides are organic solvents, which would interfere the detection results, thus limits the use of immunoassay [15].

To develop a method, a target recognition unit is required. Aptamers, a class of single strand oligonucleic acids, are selected by systematic evolution of ligands by exponential enrichment (SELEX) [16-18], which possesses superior advantages over traditional recognition molecules, because of their nontoxicity,

high specificity, high affinity, low cost and easier to synthesize and modify [19-22]. According to recent researches, highly specificity aptamers for diverse target substances (proteins [23], breast cancer [24], exsomes [25], cells [26,27], ions [28,29], bacterial [30], pesticides [31-32], etc.) have been screened. Hence, the aptamer based sensors (aptasensors) have been widely reported [33-34]. However, the aptasensors for small molecule targets meet more challenge because of the small molecule size and low molecular weight, which is difficult to capture [35]. Thus, there are relatively few reports on small molecule aptasensors. Nevertheless, the researchers devoted themselves to the study of easier sensors and high specificity aptamer [36-38]. 
Gold nanoparticles (AuNPs), have many advantages in the field of biochemical analysis because of their unique optical properties, high surface-to-volume ratio, high molar absorption coefficient, biocompatibility, non-toxicity and easy to prepare [39-41]. Among diverse AuNPs based biosensor systems, colorimetric biosensing has been widely applied for their simplicity, low cost, visible color changes, which can be considered as an alternative for on-site detection [42-44]. The ssDNAs can bind to negatively charged AuNPs through electrostatic force, thus serve as the stabilizers of AuNPs [45], in the presence of targets, the formations of ssDNA-targets would avoid adsorption from ssDNA to the surface of AuNPs, hence, the bare AuNPs immediately aggregate at high salt solution. Based on this mechanism, the AuNPs based colorimetric aptasensor become more popular [46-48].

In this article, we developed a label-free AuNPs-based colorimetric aptasensor for Chl detection, which is simple to operate and stable in organic solvents or solution containing matrix compnents. Fig. 1 is the schematic illustration for $\mathrm{Chl}$ detection. The Chl-aptamer, which was selected using ssDNA library immobilized SELEX [49], could stabilize the AuNPs in an optimal concentration of salt solution. In the presence of $\mathrm{Chl}$, the specific Chl-aptamer will bound with $\mathrm{Chl}$ first, thus the exposure to certain salt solution could cause the AuNPs self-aggregate and a color change from red to blue. Besides, the established colorimetric aptasensor was used to detect tap water, cucumber and cabbage samples to validate and evaluate the accuracy as well as practical application. The tested results were quilt satisfied demonstrating the potential use of the fabricated aptasensor to Chl capture and detection in aqueous solution and real samples.

All glasswares for preparation of AuNPs were dipped thoroughly in aquaregia (3:1 (v/v) $\left.\mathrm{HNO}_{3}-\mathrm{HCl}\right)$ for 24 hours, then washed with double distilled water and dried for use. AuNPs with a diameter around $13.0 \mathrm{~nm}$ were synthesized by means of the classical citrate reduction method [50] with a slightly improvement. Briefly, $25 \mathrm{~mL}$ of $\mathrm{HAuCl}_{4}(0.01 \%(\mathrm{w} / \mathrm{v})), 0.8 \mathrm{~mL}$ of $1 \%(\mathrm{w} / \mathrm{v})$ fresh trisodium citrate were mixed thoroughly in the bottle. Subsequently, the bottle was put into the oven at $100{ }^{\circ} \mathrm{C}$ for 2 hours. Concentration of AuNPs was calculated using the following formula $C=A /(\varepsilon \cdot b)$, where $C$ is the concentration of AuNPs, $A$ is the UV-Vis absorbance of AuNPs at $520 \mathrm{~nm}, \varepsilon$ equals to $2.7 \times 10^{8} \mathrm{M}^{-1} \mathrm{~cm}^{-1}$, is the extinction coefficients of $13.0 \mathrm{~nm}$ AuNPs, $b$ is the thickness of the measurement cuvette. To characterize the dispersion of AuNPs aqueous, UV-Vis spectra and transmission electron microscopy (TEM) images were acquired.

To fabricate a Chl colorimetric aptasensor, $25 \mu \mathrm{L}(1 \mu \mathrm{mol} / \mathrm{L})$ of Chl-aptamer, $100 \mu \mathrm{L}(3.1 \mathrm{nmol} / \mathrm{L})$ of AuNPs and $\mathrm{Chl}$ with different concentrations were evenly mixed and incubated for $30 \mathrm{~min}$ to let the target pesticide binding with aptamer completely. Later, $35 \mu \mathrm{L}(500 \mathrm{mmol} / \mathrm{L})$ of $\mathrm{NaCl}$ was added to above mixture and further diluted with deionized water to $250 \mu \mathrm{L}$. After incubation for $15 \mathrm{~min}$, the solutions were characterised by UV-Vis spectrum, and the absorbance wavelength was measured in 400-700 nm. The absorbances at $650 \mathrm{~nm}\left(A_{650}\right)$ and $520 \mathrm{~nm}\left(A_{520}\right)$ were recorded. The ratio of $A_{650} / A_{520}$ was given. All the conditions were at room temperature.

Then, a wide range of $\mathrm{Chl}$ concentration from $50 \mathrm{nmol} / \mathrm{L}$ to $10 \mu \mathrm{mol} / \mathrm{L}$ was used to test the sensitivity of the fabricated colorimetric aptasensor. The specificity of the aptasensor was determined with different 
pesticides (dimethoate, dichlorphos, carbofuran, malathion, profenofos), respectively. Besides, a mixture containing all other pesticides as well as Chl was also tested. The concentration of Chl was $1 \mu \mathrm{M}$, while the other pesticides were $10 \mu \mathrm{mol} / \mathrm{L}$.

In order to validate and evaluate the accuracy as well as practical application of the constructed chlorpyrifos colorimetric aptasensor, the cucumber, cabbage and tap water samples were tested. Tap water was collected from our laboratory, the cucumber and cabbage were purchased from the local market. The pre-treatment procedure details are as follows: tap water was filtered with a $0.22 \mu \mathrm{m}$ membrane (Millipore), then spiked with different concentrations of Chl. Cucumber and cabbage were washed, dried at room temperature, then sprayed with various concentrations of $\mathrm{Chl}$ and kept at room temperature for $12 \mathrm{~h}$, later the sampling stick evenly scraped the sample surface several times, and solved by $1 \mathrm{~mL}$ deionized water.

According to our previous works [49], we selected a highly binding Chl-aptamer. After eight selection rounds, a high enrichment ssDNA pool for Chl was obtained, the retention rates of each round were shown in Fig. S1 (Supporting information). We can see that with the increase in screening pressure, the retention rate of positive selection reaches $4.49 \%$ after the eighth round. The secondary structure of the selected Chl-aptamer was shown in Fig. S2 (Supporting information), which is composed of stem-loop and hairpin structures. The $\mathrm{K}_{\mathrm{d}}$ curve of the Chl-aptamer was shown in Fig. S3 (Supporting information), showing that the Chl-aptamer has a low dissociation constant $\left(K_{d}=58.59 \pm 6.08 \mathrm{nmol} / \mathrm{L}\right)$, which means highly binding to Chl.

The experiment conditions are important for developing a sensitive colorimetric aptasensor, such as the concentration of $\mathrm{NaCl}$, the incubation time between $\mathrm{Chl}$ and aptamer, and the incubation time of $\mathrm{NaCl}$ [38]. As shown in Fig. S4A (Supporting information), the concentrations of $\mathrm{NaCl}$ from $20 \mathrm{mmol} / \mathrm{L}$ to 100 $\mathrm{mmol} / \mathrm{L}$ were tested. The $\mathrm{A}_{650} / \mathrm{A}_{520}$ value of AuNPs solution increased with the increasing in $\mathrm{NaCl}$ concentration, while the $A_{650} / A_{520}$ value of AuNPs with aptamer solution almost keep the same during the concentration of $\mathrm{NaCl}$ increasing to $70 \mathrm{mmol} / \mathrm{L}$, which means the proper concentration of $\mathrm{NaCl}$ could not aggregate the aptamer stabilized AuNPs, but a higher concentration of $\mathrm{NaCl}$ could. Besides, for the AuNPs in the system with or without aptamer, the difference of $A_{650} / A_{520}$ value reaches the maximum after incubating with $70 \mathrm{mmol} / \mathrm{L} \mathrm{NaCl}$. Thus, we chose $70 \mathrm{mmol} / \mathrm{L} \mathrm{NaCl}$ for further study. In the incubation time optimization experiments, $1 \mu \mathrm{M}$ Chl was used. From Fig. S4B, we can see the $A_{650} / A_{520}$ value was increased with the increase in incubation time first, then stabilized after incubating for 30 min, indicating that the binding interaction between $\mathrm{Chl}$ and aptamer reached saturation at $30 \mathrm{~min}$. From Fig. $\mathrm{S} 4 \mathrm{C}$, we can see the optimal incubation time of $\mathrm{NaCl}$ is $15 \mathrm{~min}$.

AuNPs in different systems were characterized with UV-Vis spectrum and TEM images. As shown in Fig. $2 \mathrm{~A}$, we can see that in the system of AuNPs in $70 \mathrm{mmol} / \mathrm{L} \mathrm{NaCl}$, the $A_{520}$ hugely decreased and the $A_{650}$ increased (red line), meaning the aggregation of AuNPs. The Chl-aptamer with random coil structure can be adsorbed on the surface of AuNPs by electrostatic interaction thus can protect AuNPs from ioninduced aggregation and, therefore, AuNPs are more stable in salt solution [51]. Once the AuNPs were 
incubated with aptamer (purple line), the proper concentration of $\mathrm{NaCl}$ could not make the AuNPs aggregate, which means the Chl-aptamer could stabilize the AuNPs dispersion. In the presence of Chl, the strong interaction between $\mathrm{Chl}$ and aptamer could make the conformation of aptamer change, the formation of aptamer-target complex could avoid the adsorption from aptamer to the surface of AuNPs. Hence, the unprotected AuNPs would aggregate in salt solution and the extinction peak was shifted to a longer wavelength. Thus, a decrease of $A_{520}$ and an increase of $A_{650}$ were observed, leading an increase in $A_{650} / A_{520}$ (blue line). As mentioned in the study of Sato and co-workers [52], this aggregation phenomenon is driven by the London-van der Waals attractive force between AuNPs, when the repulsive interaction is greatly reduced by the formation of duplexes on their surface. The attractive force works from a distance and leads to the aggregation. Meanwhile, Fig. 2B to Fig. 2E are the corresponding TEM images of each systems.

Subsequently, the label-free AuNPs based colorimetric aptasensor was established for Chl standard solution ( 0 to $10 \mu \mathrm{mol} / \mathrm{L})$ analysis. The UV-Vis spectra were shown in Fig. 3A, we can see that with the increase in Chl concentration, the $A_{520}$ value kept decreasing, while the $A_{650}$ value also kept increasing. The scatterplot of the $A_{650} / A_{520}$ values with different concentrations of Chl was shown in Fig. 3B, it was shown that the $A_{650} / A_{520}$ value increased significantly at the concentration of Chl from 0 to $5 \mu \mathrm{mol} / \mathrm{L}$, and slowed down after the $\mathrm{Chl}$ concentration kept increasing to $10 \mu \mathrm{mol} / \mathrm{L}$. It is worth mentioning that there are two linear relationships between $A_{650} / A_{520}$ value and the concentration of Chl. At low concentration range of $\mathrm{Chl}$ (ranging from $50 \mathrm{nmol} / \mathrm{L}$ to $200 \mathrm{nmol} / \mathrm{L}$ ), the linearization equation is $\mathrm{y}=$ $0.102+9.126 \times 10^{-4} \mathrm{C}\left(\mathrm{R}^{2}=0.996\right)(\mathrm{Fig} .3 \mathrm{C})$, while at high concentration range of $\mathrm{Chl}$ (ranging from 200 $\mathrm{nmol} / \mathrm{L}$ to $5000 \mathrm{nmol} / \mathrm{L})$, the linearization equation is $\mathrm{y}=0.266+1.124 \times 10^{-4} \mathrm{C}\left(\mathrm{R}^{2}=0.997\right)$ (Fig. 3D). The limit of detection (LOD) is as low as $14.46 \mathrm{nmol} / \mathrm{L}$, which is given by the equation: $L O D=3 \times \mathrm{S}_{B} / \mathrm{b}$, $\left(S_{B}\right.$ is the standard deviation of twenty independent blank samples and $b$ is the sensitivity of the calibration graph.) [29]

The specificity of the fabricated colorimetric aptasensor was evaluated by comparing the $A_{650} / A_{520}$ value of solutions containing $1 \mu \mathrm{mol} / \mathrm{L}$ chlorpyrifos with other pesticides at concentrations 10 times higher, such as dimethoate, dichlorphos, carbofuran, malathion, and profenofos. A water solution was used as the blank. As shown in Fig. 4 , the $A_{650} / A_{520}$ value showed the ability of the current aptasensor to specifically detect chlorpyrifos even in a mixture, while a negligible change of $A_{650} / A_{520}$ value in detecting other pesticides. The result indicates this aptasensor could specifically detect chlorpyrifos among other pesticides.

The sample detection results were shown in Table 1. It was confirmed that the developed colorimetric aptasensor displays excellent capability for the accurate detection of chlorpyrifos in tap water, cucumber and cabbage samples. The excellent recovery values with acceptable RSD values below $5 \%$ demonstrates that the proposed aptasensor could be applied to detecet chlorpyrifos in real samples. 
Table 1 Determination of $\mathrm{Chl}$ in real samples

\begin{tabular}{ccccc}
\hline Sample & Added amount (nmol/L) & Found amount ${ }^{\mathrm{a}}(\mathrm{nmol} / \mathrm{L})$ & Recovery (\%) & $\begin{array}{c}\text { RSD b } \\
(\mathrm{n}=5, \%)\end{array}$ \\
\hline Tap water & 100.0 & 105.6 & 105.6 & 2.8 \\
& 500.0 & 480.8 & 96.2 & 1.6 \\
\cline { 2 - 5 } Cucumber & 1000.0 & 1032.2 & 103.2 & 2.2 \\
\cline { 2 - 5 } & 100.0 & 98.3 & 98.3 & 2.5 \\
\cline { 2 - 5 } & 500.0 & 509.2 & 101.8 & 1.8 \\
\hline Cabbage & 1000.0 & 988.7 & 98.9 & 2.3 \\
& 100.0 & 103.6 & 98.1 & 3.2 \\
\cline { 2 - 5 } & 500.0 & 490.5 & 97.1 & 4.8 \\
\hline
\end{tabular}

a Mean values of three determinations.

b Standard deviation

In summary, we fabricated a label-free AuNPs based colorimetric aptasensor for sensitive detection of Chl. The Chl-aptamer was obtained using ssDNA library immobilized streptavidin-magnetic beads SELEX technique. The fabricated colorimetric aptasensor exhibits excellent sensitivity for Chl detection with the limit of detection as low as $14.46 \mathrm{nmol} / \mathrm{L}$. Furthermore, the real samples detection showed satisfying results with excellent recovery values between $96.2 \%$ and $105.6 \%$ and acceptable RSD values below $5 \%$, demonstrating the developed aptasensor can serve as a promising candidate for Chl detection in the area of biosensors, which also showed a great potential in simple, cheap and rapid detection of $\mathrm{Chl}$.

\section{Declarations}

\section{Acknowledgment}

This work was financially supported by the National Key Research and Development Program of China (2018YFC1602905), the National Natural Science Foundation of China (61871180 and 61527806) for the financial supports.

\section{References}

1. S.Y. Foong, N.L. Ma, S.S. Lam, et al., J. Hazard. Mater. 400 (2020) 123006.

2. X. Huang, H. Cui, W. Duan, Ecotox. Environ. Safe. 200 (2020) 110731.

3. A.P. Mestre, P.S. Amavet, I.S. Sloot, et al., Chemosphere 252 (2020) 126433.

4. X. Zhang, W. Cui, K. Wang, et al., Environ. Res. 188 (2020) 109785.

5. S.W. Todd, E.W. Lumsden, Y. Aracava, et al., Neuropharmacology 180 (2020) 108271.

6. M.Y. Xu, P. Wang, Y.J. Sun, et al., Chem. Res. Toxicol. 32 (2019) 122-129.

7. M. Lasagna, M.S. Hielpos, C. Ventura, et al., Environ. Safe. 205 (2020) 111312.

8. P. Moyano, J. Garcia, J.M. Garcia, et al., Chemosphere 251 (2020) 126426.

9. J. Zhang, L. Liu, L. Ren, et al., J. Hazard. Mater. 334 (2017) 121-131. 
10. L. He, X. Luo, H. Xie, et al., Anal. Chim. Acta 655 (2009) 52-59.

11. F. Darvishnejad, J.B. Raoof, M. Ghani, Anal. Chim. Acta 1140 (2020) 99-110.

12. P. Balsini, H. Parastar, J. Sep. Sci. 42 (2019) 3553-3562.

13. Y. Lee, Y.J. Kim, M.S.I. Khan, et al., J. Sep. Sci. 42 (2020) 4047-4057.

14. C. Zhai, T.F. Xu, Y.K. Peng, et al., Spectrosc. Spect. Anal. 36 (2016) 2835-2840.

15. L. Fan, G. Zhao, H. Shi, M. Liu, Z. Li, Biosens. Bioelectron. 43 (2013) 12-18.

16. H. Wang, H. Cheng, J. Wang, et al., Talanta 154 (2016) 498-503.

17. A. Sinha, P. Gopinathan, Y.D. Chung, et al., Biosens. Bioelectron. 122 (2018) 104-112.

18. Y. Luo, J. Wang, L. Yang, et al., Sens. Actuators B Chem. 276 (2018) 128-135.

19. R. Huang, L. He, S. Li, et al., Nanoscale 12 (2020) 2445-2451.

20. Z.K. Guo, Y. Liu, N.Y. He, et al., Chin. Chem. Lett. 32 (2021) 40-47.

21. Z. Xi, R. Huang, Z. Li, et al., ACS Appl. Mater. Inter. 7 (2015) 11215-11223.

22. M. Liu, Z. Yang, B. Li, et al., Food Chem. 339 (2021) 128059.

23. H. Li, S. Xing, J. Xu, et al., Talanta 221 (2021) 121670.

24. M. Liu, L. Xi, T. Tan, et al., Chin. Chem. Lett. 32 (2021) 1726-1730.

25. R.R. Huang, L. He, Y.Y. Xia, et al., Small 15 (2019), 1900735.

26. Z. Guo, C. Wang, S. Li, et al., J. Nanosci. Nanotechnol. 20 (2020) 3373-3377.

27. T. Li, J. Yang, Z. Ali, et al., Sci. China Chem. 60 (2017) 370-376.

28. J. Pan, Q. Li, D. Zhou, et al., Talanta 189 (2018) 370-376.

29. Y. Liu, Y.X. Lai, G.J. Yang, et al., J. Biomed. Nanotechnol. 13 (2017) 1253-1259.

30. S. Chen, X. Yang, S. Fu, et al., Food Control 115 (2020) 107281.

31. M. Jiang, C. Chen, J. He, et al., Food Chem. 307 (2020) 125534.

32. Y. Qi, Y. Chen, F. Xiu, et al., Sens. Actuators B Chem. 304 (2020) 127359.

33. H. Shen, W. Deng, Y. He, et al., Biosens. Bioelectron. 168 (2020) 112516.

34. Y. Yang, Y. Yin, X. Li, et al., Sens. Actuators B Chem. 319 (2020) 128250.

35. Q. Ma, Y. Wang, J. Jia, et al., Food Chem. 249 (2018) 98-103.

36. N. Duan, S. Qi, Y. Guo, et al., LWT-Food Sci. Technol. 134 (2020) 110017.

37. M. Liu, A. Khan, Z. Wang, et al., Biosens. Bioelectron. 130 (2019) 174-184

38. N. Duan, S. Wu, X. Chen, et al., Food Chem. 61 (2013) 3229-3234.

39. J. Yi, P. Wu, G. Li, et al., Microchim. Acta 186 (11) (2019) 711.

40. L. He, R.R. Huang, P.F. Xiao, et al., Chin. Chem. Lett. 32 (2021) 1593-1602.

41. H. Shi, G. Zhao, M. Liu, et al., J. Hazard. Mater. 260 (2013) 754-761.

42. A.N. Berlina, A.V. Zherdev, S.M. Pridvorova, et al., J. Nanosci. Nanotechnol. 19 (2020) 5489-5495.

43. K. Abnous, N.M. Danesh, M. Ramezani, et al., Microchim. Acta 185 (2018) 216. 
44. J. Jia, S. Yan, X. Lai, et al., Food Anal. Method. 11 (2018) 1668-1676.

45. Y. Liu, T. Li, C. Ling, et al., Chin. Chem. Lett. 30 (2019) 2359-2362.

46. C. Xu, Y. Ying, J. Ping, Microchim. Acta 186 (2019) 448.

47. M. Qi, C. Tu, Y. Dai, et al., Anal. Methods 10 (2018) 3402-3407.

48. L. Qiao, H. Wang, J. He, et al., Food Chem. 340 (2021) 128181.

49. Y. Liu, G.J. Yang, T.T. Li, et al., Chin. Chem. Lett. 32 (2021) 1957-1962.

50. G. Frens, Nature Phys. Sci. 241 (1973), 20-22.

51. Y.S. Kim, J.H. Kim, I.A. Kim, et al., Biosens. Bioelectron. 26 (2010) 1644-1649.

52. K. Sato, K. Hosokawa, M. Maeda, J. Am. Chem. Soc. 125 (2003) 8102-8103.

Figures

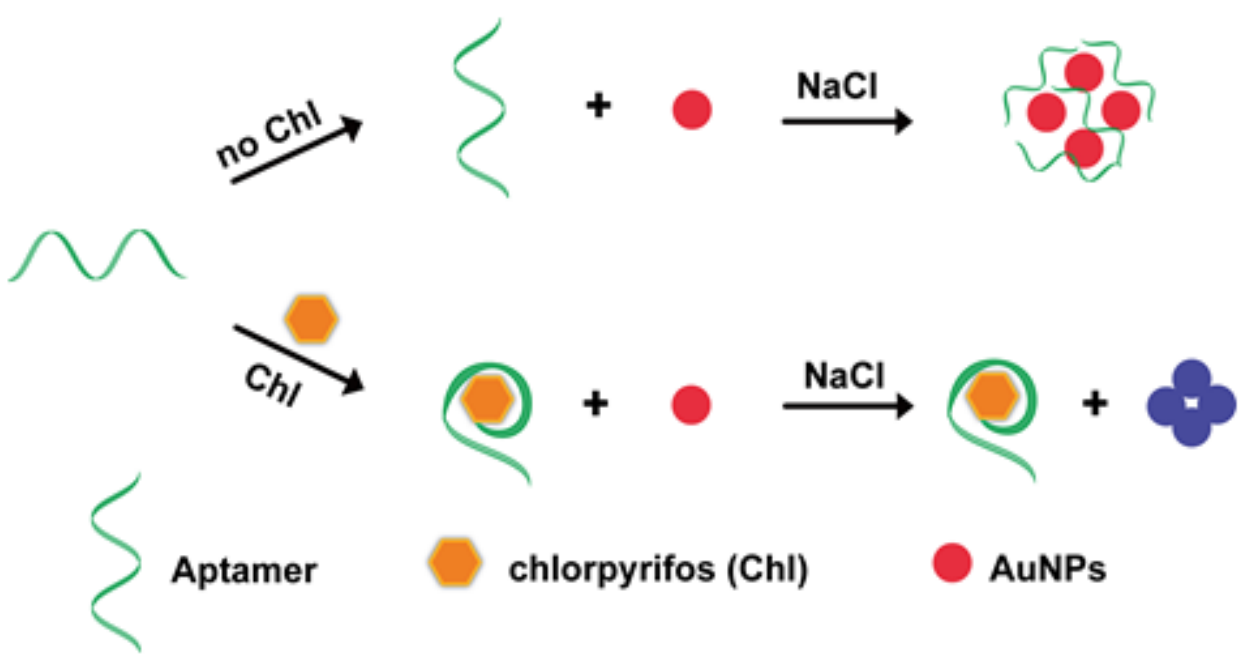

Figure 1

Schematic illustration of the fabricated colorimetric aptasensor for chlorpyrifos detection. 

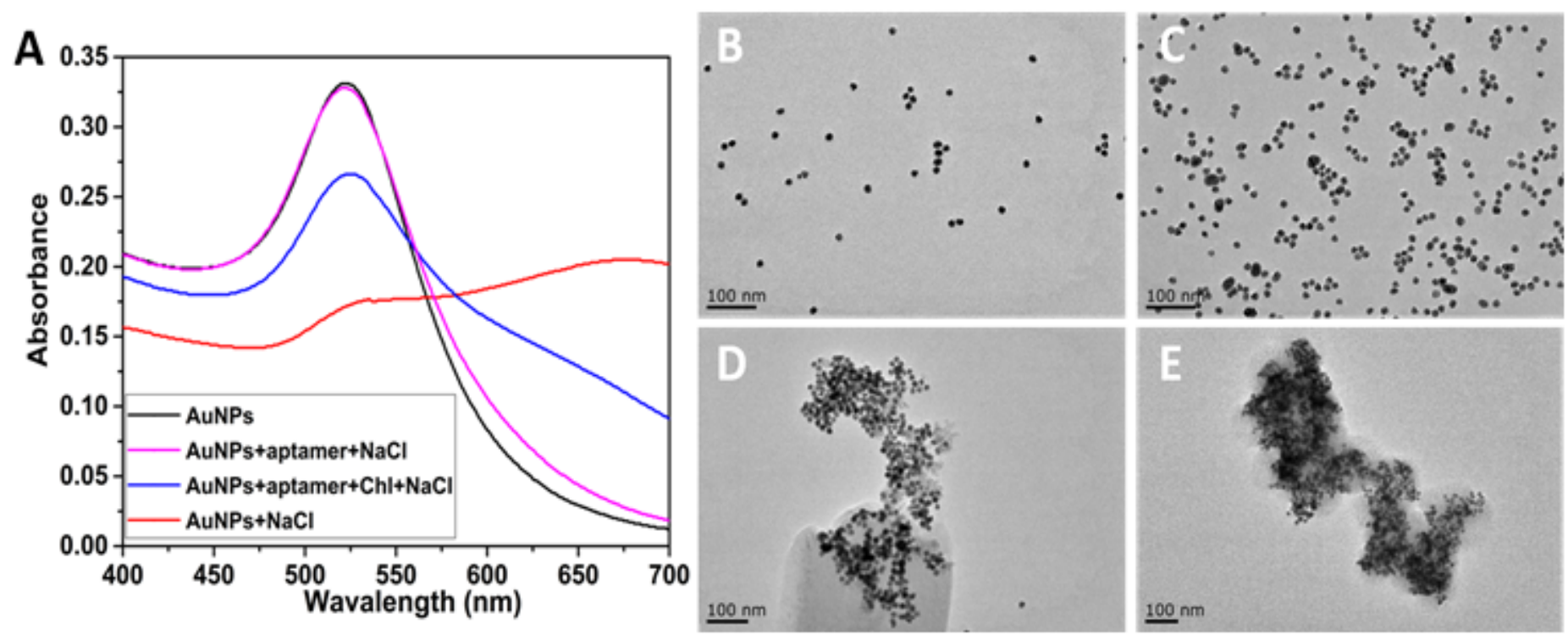

Figure 2

(A) UV-Vis spectra of AuNPs in different systems. From up to down is AuNPs (black line), AuNPs+aptamer+ $\mathrm{NaCl}$ (purple line), AuNPs+aptamer+Chl+NaCl (blue line), AuNPs+ $\mathrm{NaCl}$ (red line), respectively. TEM images of AuNPs in different systems. (B) AuNPs, (C) AuNPs+aptamer+NaCl, (D) AuNPs+aptamer+Chl+NaCl, (E) AuNPs+NaCl.
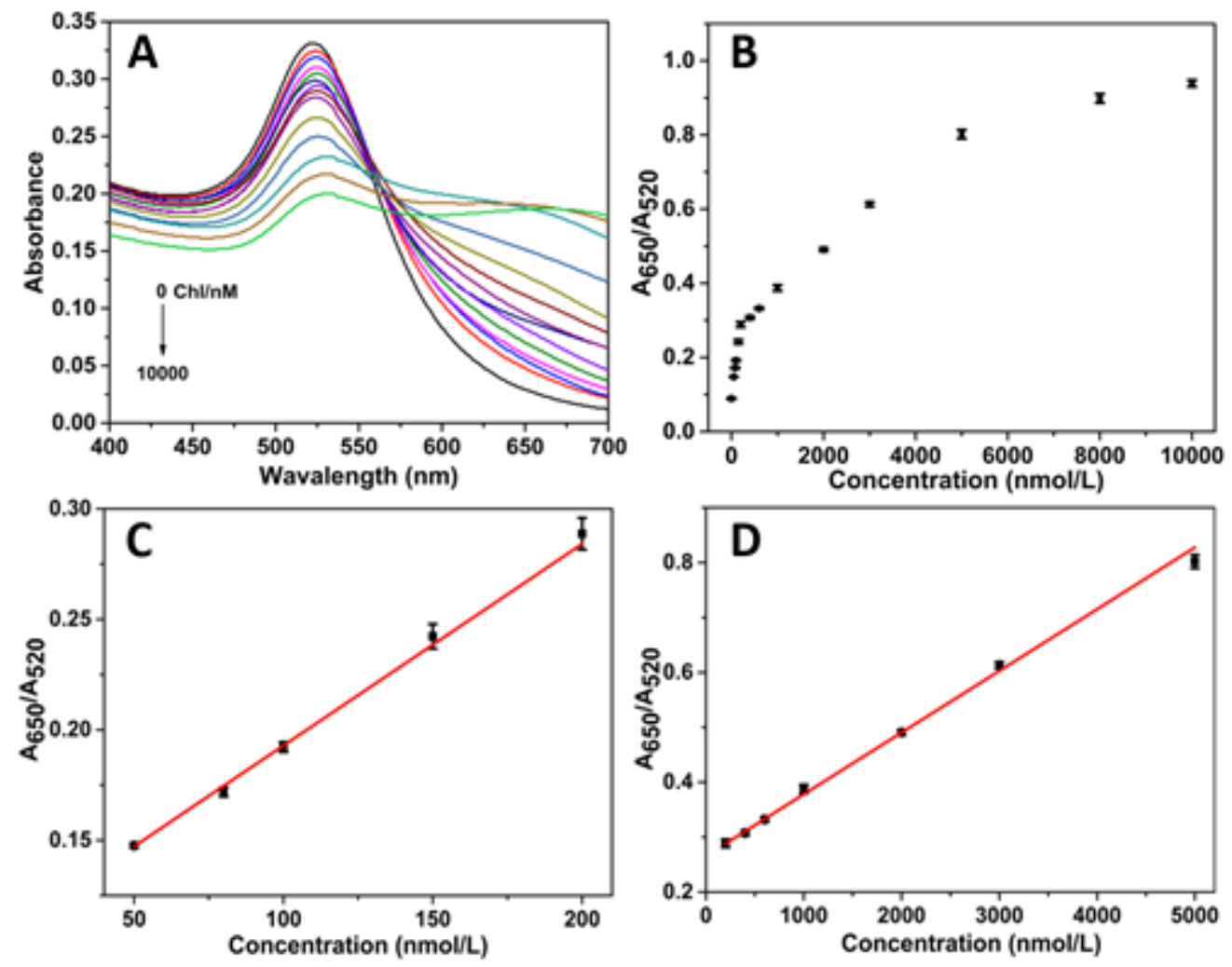

Figure 3 
(A) UV-Vis spectra of different concentration of $\operatorname{Chl}(0,50,80,100,150,200,400,600,1000,2000,3000$, $5000,8000,10000 \mathrm{nmol} / \mathrm{L}$ ); (B) Scatterplot of $A_{650} / A_{520}$ value at different concentration of Chl; (C) The linear relationship between the $A_{650} / A_{520}$ value and low concentration of $\mathrm{Chl}(50-200 \mathrm{nmol} / \mathrm{L})$; (D) The linear relationship between the $A_{650} / A_{520}$ value and high concentration of $\mathrm{Chl}(200-5000 \mathrm{nmol} / \mathrm{L})$.

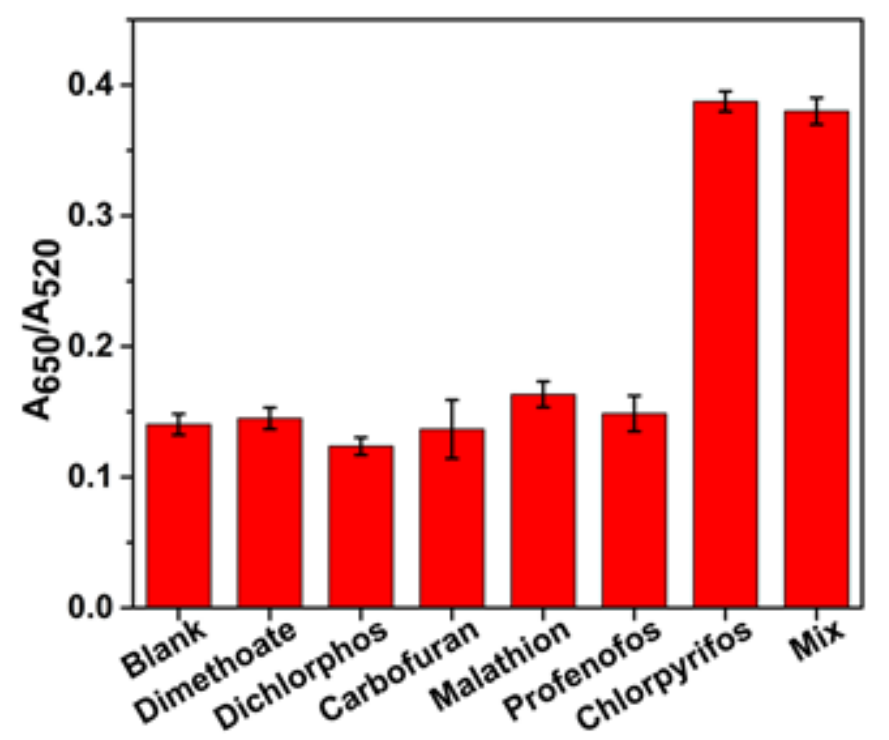

\section{Figure 4}

The selectivity of the proposed colorimetric aptasensor, Blank means without any pesticide; Mix means the solution contains $1 \mu \mathrm{M} \mathrm{Chl}$ and $10 \mu \mathrm{M}$ of dimethoate, dichlorphos, carbofuran, malathion, profenofos, respectively.

\section{Supplementary Files}

This is a list of supplementary files associated with this preprint. Click to download.

- revised2SIEH.doc

- GraphicalAbstract.docx 